\title{
Rats acquire win-stay more readily than win-shift in a water escape situation
}

\author{
LARRY W. MEANS \\ East Carolina University, Greenville, North Carolina
}

\begin{abstract}
Independent groups of Sprague-Dawley rats were found to acquire a win-stay, but not a win-shift, escape response in a circular water maze in each of three experiments that varied with respect to swim time and distance prior to escape following an incorrect first choice. The subjects were given pairs of trials: an information trial and a test trial, separated by $10 \mathrm{~min}$. On the information trial the camouflaged escape platform was randomly placed in one of two positions. On the test trial the platform was placed in the same position for the win-stay task and in the opposite position for the win-shift task. Animals that did not acquire either the stay or the shift response perseverated in their responses, consistently going to the same escape location first on both information and test trials. In the fourth experiment, in which win-shift, win-stay, and perseveration all led to escape, all rats perseverated in their responses. It was concluded that response perseveration and win-stay are more natural responses than win-shift for rats in a water escape situation. This finding contrasts with the spontaneous alternation and readily acquired win-shift behavior previously demonstrated in rodents in exploratory and appetitive situations.
\end{abstract}

When unstressed normal rodents are given two consecutive trials in a T-maze, they typically alternate choices (Dember \& Earl, 1957; Means, Harrell, Mayo, \& Alexander, 1974; Means, Leander, \& Isaacson, 1971), a phenomenon called spontaneous alternation. Accordingly, normal rats readily acquire a food-reinforced alternation (win-shift) response (Means et al., 1971). Also, when given a choice, they typically enter a novel, previously unavailable alley rather than an alley in which they have been repeatedly reinforced (Means et al., 1974). Furthermore, in a study comparing acquisition of win-stay and win-shift responses during pairs of trials in a T-maze in which the animal was forced to a randomly selected goal box on the first trial and given a choice on the second trial, it was demonstrated that rats are capable of acquiring either response, but that it takes fewer trials to acquire the win-shift response (Stanton, Thomas, \& Brito, 1984). Also, in the radial-arm maze rats spontaneously enter novel (unentered) alleys and more readily learn to enter previously unentered arms (win-shift) than to return to arms that have already been entered (win-stay), even if each arm is baited with food or water before all trials (Gaffan \& Davies, 1981; Olton \& Schlosberg, 1978). These seemingly paradoxical observations (animals' selecting relatively novel or nonreinforced alleys over familiar and/or previously reinforced alleys) have been attributed to the notion that animals seek optimal levels of arousal (Berlyne, 1960; Dember \& Earl, 1957; Glanzer, 1958). More recently, Gaffan and Davies (1981) also presented an arousal explanation of spontaneous alternation, in which it is assumed that alternation increases

I gratefully acknowledge the assistance of Tracy R. Comer and Margaret F. O'Neal in the preparation of this manuscript. Address correspondence to Larry W. Means, Psychology Department, East Carolina University, Greenville, NC 27858-4353. arousal. Under conditions of relatively low arousal, such as an exploratory situation, an animal will alternate to increase arousal. Under conditions of high arousal, such as an escape situation, an animal will perseverate to avoid increasing its arousal level. Olton and his colleagues $(\mathrm{Ol}-$ ton, Handelmann, \& Walker, 1981; Olton \& Schlosberg, 1978) attributed the animals' preference for win-shift over win-stay responses in the radial-arm maze to the influence of species-specific foraging activities (a tendency to avoid locations where food has been exhausted).

Although the optimal-arousal-level hypothesis predicts that in a stressed situation animals will tend to perseverate in their responses, ethological considerations could lead to a prediction of either perseveration or alternation. Inasmuch as novel stimuli are assumed to be arousing (Berlyne, 1960; Dember \& Earl, 1957; Glanzer, 1958), a stressed or highly aroused animal would be expected to avoid novel environmental locations, including novel or relatively novel alleys in a maze. In fact, shocking mice in the startbox, choice point, or choice alley of a T-maze results in the animals' developing a perseverative response, always going to the same choice alley. This is true even when making an alternation (win-shift) response leads to shock avoidance (Mitchell, Koleszar, \& Scopatz, 1984). From an ethological viewpoint, an animal attempting to escape a predator or other threat would be more likely to return to a familiar "safe" place than to explore novel places in the environment; however, it may be argued that, because of the unpredictable nature of the environment, it is advantageous for rats to have many safe places and to alternate between them (Burešová, Bureš, Oitzl, \& Zahalka, 1985).

In water escape tasks, rats tend to return on the second trial to the location in which an escape platform had been found on the first trial (Morris, 1984). Many studies have 
shown that rats readily acquire this win-stay escape response in the circular water maze under a variety of training conditions, even when they start from different locations on successive trials (Morris, 1981, 1984; Sutherland \& Linggard, 1982; Sutherland, Whishaw, \& Kolb, 1983).

Recently, Burešová et al. (1985) reported that rats acquired a win-shift response in a radial-arm water escape maze. However, the unique procedures used by Burešová et al. make comparison with other water escape tasks difficult. Since the escape bench found at the end of an arm was lowered $15 \mathrm{sec}$ after a subject reached it, that arm became, in essence, an "unsafe" place. The rats in this study were actually performing a series of escapes during each trial. More recently, Mactutus and Murray (1986) reported that in the circular water maze rats acquired a win-stay response when the escape platform location was held constant and a win-shift response when the platform was randomly placed in one of four positions. However, the use of latency as a response measure may have yielded misleading results. Latency provides no information concerning the first choice of the subjects; the fact that latencies for both win-stay (fixed) and win-shift (random) groups decreased across trials was used to infer learning of the appropriate strategy. It is likely that the random group, rather than learning a win-shift rule per se, simply learned to swim rapidly in a path containing all four platform locations. Thus, what appeared to be a win-shift strategy might actually have been a perseverative response.

Although reference memory (recalling those things that remain constant over trials, such as a win-stay rule) is involved in all multiple-trial tasks, working memory (recalling those things that are unique to a given trial, such as the location of the escape platform on the immediately preceding trial) probably is and certainly can be made an essential feature of all of the above tasks. Douglas (1966) found that rats in a T-maze spontaneously alternated at above-chance levels when odor trails were eliminated, making working memory essential. Likewise, Olton and Collison (1979) found that the win-shift response of rats in the radial-arm maze was not dependent on odor trails. Of course, water mazes are assumed to be free of cues that would provide information to a subject about a specific preceding response.

The present series of experiments was designed to compare rats' acquisition of win-stay and win-shift responses in an aversive situation in which working memory is required. It was hypothesized that the tendency of rodents to perseverate in their responses in an aversive situation would make acquisition of win-stay responses easier than acquisition of win-shift responses. Thus, rats' acquisition of free-choice win-stay and win-shift responses in a circular water maze was examined.

\section{GENERAL METHOD}

\section{Subjects}

The subjects were male Sprague-Dawley rats from the East Carolina University Psychology Department colony, which is maintained in accordance with the conditions prescribed by the American Association for Accreditation of Laboratory Animal Care. The rats were 90-120 days old at the beginning of the experiments. They were housed individually in $35 \times 20 \times 20 \mathrm{~cm}$ stainless steel wiremesh cages and had free access to water and lab chow. The room was maintained at $24^{\circ} \pm 2^{\circ} \mathrm{C}$ with a 16:8-h light:dark cycle in which the lights came on at $0700 \mathrm{~h}$.

\section{Apparatus}

The behavioral testing apparatus was a circular metal pool $62 \mathrm{~cm}$ tall and $140 \mathrm{~cm}$ in diameter. The pool was placed against a long wall in a rectangular room with two solid walls, a wall with a row of windows covered with Venetian blinds, and a wall with a distinct door; thus, prominent extramaze cues were available. The pool was marked into radial thirds by clear fishing line strung $45 \mathrm{~cm}$ above the surface of the water. A position at the center of the arc forming the perimeter of one section served as the starting point for all trials. The pool was filled with water to a level of $34 \mathrm{~cm}$, $1 \mathrm{~cm}$ above the surface of an escape platform. The water was clouded with nontoxic white paint to camouflage the platform and was maintained at room temperature. A tablespoon of Clorox was added to the water daily as an antibacterial agent, and the water was changed every 4 to 5 days.

\section{Procedure}

Experiments 1, 2, and 3 involved comparing groups of rats on the acquisition of win-stay and win-shift escape responses. Training consisted of giving the subjects pairs of free-choice trials. For each pair of trials the rats were placed individually into plastic transfer cages and taken in squads of 6 to the adjoining room for testing. Each pair of trials consisted of an information trial and a test trial. The test trial was initiated $10 \mathrm{~min}$ after the information trial. The subjects remained in the plastic transfer cages between trials within a pair. On each trial the rat was placed in the water at the starting position and allowed to swim until it reached the escape platform. During this time, the experimenter remained behind the starting point. The rat was removed from the pool as soon as it had climbed onto the platform. Any rat that failed to find the platform within $5 \mathrm{~min}$ was removed from the water.

During an information trial the escape platform was placed in the section of the pool either to the left or to the right of the starting section (the left or right choice section), according to a Gellermann-type sequence (Gellermann, 1933). Each set of 20 pairs of trials had 10 right and 10 left correct choices, and the correct choice was never on the same side for more than 3 consecutive pairs of trials. For the group being trained on win-stay, the escape platform was placed in the same section of the pool on the test trial as it had been during the information trial of the pair; for the group being trained on win-shift, the escape platform was placed in the opposite section of the pool on the test trial. Upon completion of a pair of trials the rats were returned to their home cages.

\section{EXPERIMENT 1 WIN-STAY VERSUS WIN-SHIFT}

The first experiment was conducted to compare rats on the acquisition of win-stay and win-shift water escape responses. The rats were placed in the pool and allowed to swim until they escaped onto the platform.

\section{Method}

Subjects. Twenty rats, 10 in the win-stay group and 10 in the win-shift group, were used in this experiment.

Procedure. Prior to training the rats were handled in groups of 5 for approximately $10 \mathrm{~min}$ twice a day for 4 days. Training consisted of giving each rat two pairs of trials per day, Monday through 
Friday, for 2 weeks, for a total of 20 pairs of trials. On each trial a circular white plastic escape platform $(21 \mathrm{~cm}$ in diameter) was placed on the midpoint of the radius bisecting the left or right choice section of the pool. One pair of trials was given at approximately $0900 \mathrm{~h}$ and one at approximately $1800 \mathrm{~h}$. The first section entered and the latency to reach the escape platform were recorded on each trial.

\section{Results}

All data reported in this paper were evaluated with mixed-factors analyses of variance (ANOVAs) and a posteriori Neuman-Keuls tests, when appropriate. The number of correct first choices (i.e., the rat entered the section containing the escape platform first) on both the information and test trials is shown for both the win-stay and win-shift groups in Figure 1. A three-way (task $x$ trial type [test or information] $\times$ blocks) mixed-factors ANOVA resulted in a significant task $\times$ trial type interaction $[F(1,18)=8.459, p<.01]$ and significant task $[F(1,18)=11.977, p<.01]$ and trial type $[F(1,18)=$ $5.737, p<.05]$ main effects. Examination of Figure 1 clearly reveals that the significant differences resulted from the fact that the win-stay group made more correct choices on test trials than they did on information trials and than the win-shift group made on either type of trial. However, a posteriori tests failed to reveal any significant differences among the four task $\times$ trial type means ( $p>.05$ in each case).

To reduce skew, each latency score (total latency for four information or four test trials within a block) was transformed to the $\log (X+1)$. A three-way mixed-factors ANOVA performed on the transformed latency scores resulted in significant trial type $[F(1,18)=4.730$, $p<.05]$ and block $[F(4,72)=95.518, p<.001]$ main effects. Also, the task $x$ trial type interaction was the only interaction involving task that was significant $[F(1,18)=$

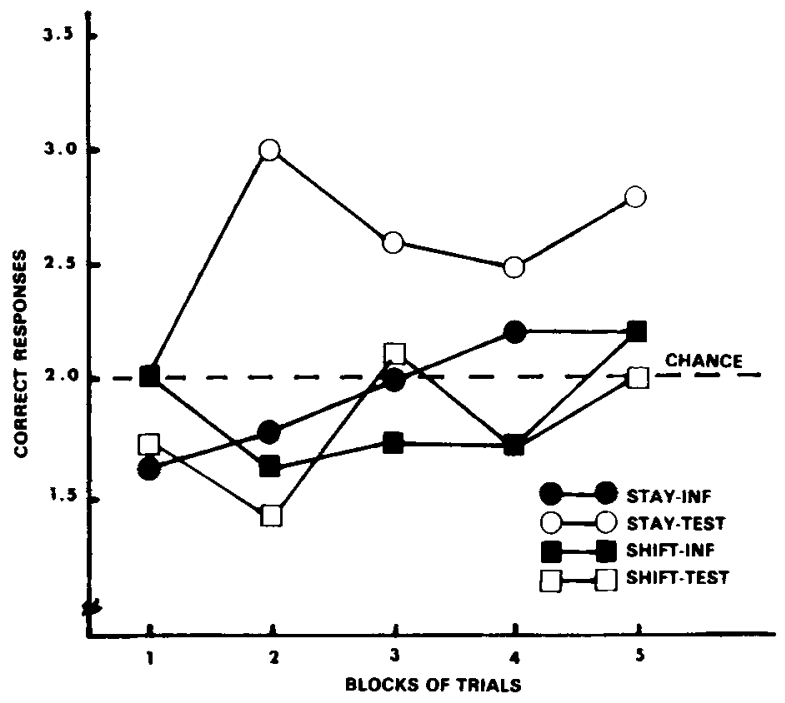

Figure 1. Mean number of correct responses made on information (INF) and test trials of win-stay and win-shin tasks across blocks of four peirs of trials. Note that 2.0 correct responses equals $50 \%$ correct choices or chance-level performance.

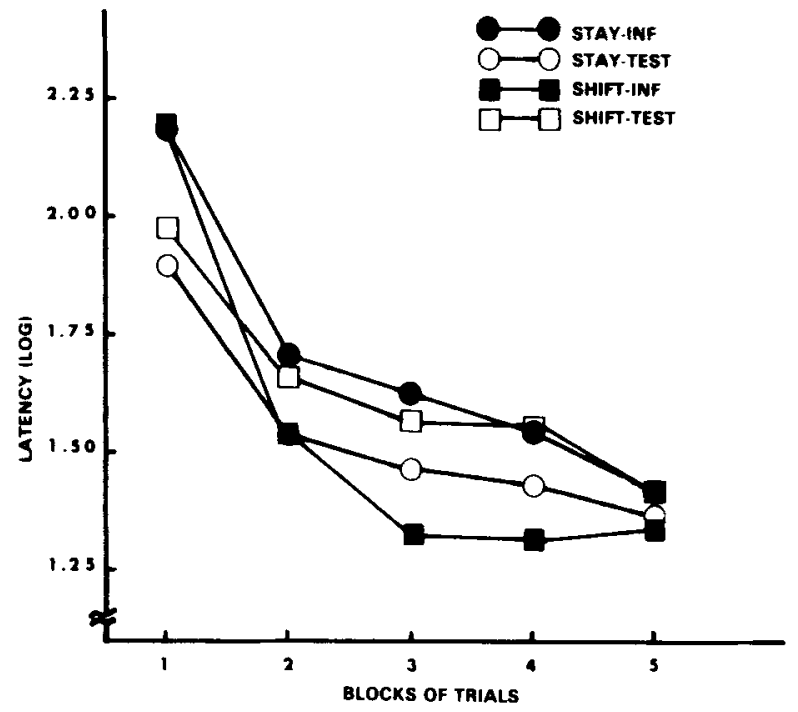

Figure 2. Mean log of totel batency for four information (INF) and four test trials within each block for the win-stay and win-shint tasks.

67.521, $p<.001$ ]. Post hoc tests revealed that the win-stay group had lower mean test-trial log latencies than either group had on information trials $(p<.05$ in each case). The mean latencies for all subjects across all information and test trials were 15.7 and $11.4 \mathrm{sec}$, respectively. Examination of Figure 2 reveals that both groups developed shorter latencies on both information and test trials across blocks of trials. Further examination of Figure 2 reveals that over the last four blocks the win-stay group had shorter latencies on the test trials than on the information trials, whereas the win-shift group had just the opposite-longer latencies on the test trials than on the information trials.

Casual examination of the first-choice raw data reveals that at times many of the subjects, particularly in the win-shift group, perseverated in making either left or right choices. These subjects went to the same platform location first on a series of consecutive trials, including both information and test trials. Thus, every seventh or greater consecutive response to the same platform position across both information and test trials was scored as a perseverative response. This criterion was based on the fact that, according to the Gellermann-type sequence used, the platform was never placed in the same position for more than three consecutive pairs of trials; thus it would never be correct for an animal to choose one location more than six consecutive times. The percentage of perseverative responses (number of actual perseverative responses/total number of responses, excluding the first six responses, which by definition could not be perseverative responses) was determined for both groups. Figure 3 shows the mean percentage of perseverative responses made by both groups over the last four blocks of four pairs of trials. The first block of four pairs of trials is not included, as it contained only two trials during which perseveration could have occurred; six consecutive same-side choices 


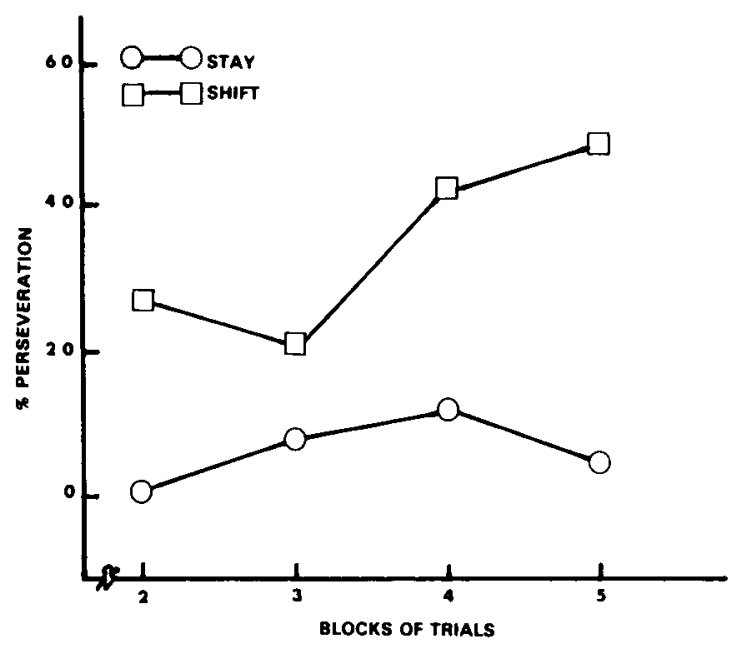

Figure 3. Mean percentage of perseverative responses made on win-stay and win-shift tasks across blocks of four pairs of trials.

are necessary before a subject can be scored as perseverating. A two-way mixed-factors ANOVA (task $\times$ block) produced significant task $[F(1,18)=4.701, p<.05]$ and block $[F(3,54)=3.289, p<.05]$ main effects and a nearly significant task $\times$ block interaction $[F(3,54)=$ $2.567, p=.06$ ]. Thus, the win-shift group made a higher percentage of perseverative responses than did the win-stay group, and both groups, but especially the win-shift group, showed an increasing percentage of perseverative responses across blocks of trials.

In summary, the win-stay group showed better performance than the win-shift group, as evidenced by more correct choices and shorter latencies on test trials and less perseverative responding on both information and test trials over the last four blocks.

\section{Discussion}

The results suggest several conclusions about the acquisition of win-stay and win-shift escape responses by rats. First, the rats acquired the win-stay response much more readily than the win-shift response, as evidenced by both the first-choice and latency scores. In fact, over the 20 pairs of trials given, only the win-stay group exceeded chance performance on test trials. Second, the rats that failed to acquire the correct response developed a perseverative response pattern, always going to the same section of the pool first on both information and test trials. Thus, the win-shift group, upon failing to acquire the shift response, showed more perseveration than the win-stay group. It should be noted that perseverating in making responses is not the same as making win-stay responses. Response perseveration involves consistently choosing first the same section selected first on the preceding trial, regardless of where the escape platform was located. On the other hand, win-stay responding involves choosing first the section where the platform was located on the immediately preceding trial. These two types of responses appear to involve the use of different mnemonic strate- gies. Response perseveration seems to involve the use of reference memory (going to the section selected on many consecutive previous trials), whereas win-stay responding seems to involve working memory (going to the section where the escape platform was located on the immediately preceding trial). Third, the rats in both groups used reference memory, learning that the escape platform was in one of two positions on any given trial. This is evidenced by their decreasing latencies across both information and test trials as they learned to avoid portions of the pool where the platform was never placed. Since it took the rats less than $2 \mathrm{sec}$ to swim from the incorrect platform location to the correct platform location, a rat's latency decreased across trials even if it made incorrect first choices. Note that if the first choice had not been recorded and the test- and information-trial latencies had not been examined separately, the apparent conclusion would have been that both the win-stay group and the win-shift group acquired their respective responses within the 20 pairs of trials. Fourth, the rats in the win-stay group used working memory to perform the task. They could perform better than chance on test trials only by using working memory, since the rats could not detect the position of the escape platform, as evidenced by the essentially chance-level performance of both groups on the information trials and of the win-shift group on the test trials.

Observation of the rats during training revealed that those rats that perseverated in their choices made very stereotypic responses. Perseverating rats would consistently swim first to the same platform position; if the platform was not in that section they would immediately go straight to the other potential location and escape. Thus, being incorrect in their first choice would delay their escape by only a few seconds. Such a response pattern reveals that these rats had learned that the escape platform was always placed in one of two locations, namely, at the center of either the left or the right choice section. However, the pattern also reveals that they (1) had not learned the win-stay or win-shift rule, whichever was applicable; (2) could not recall on test trials the location of the platform on information trials; or (3) simply responded independently of the position of the platform on the immediately preceding trial. Since making an incorrect first choice delayed the rat's escape from the water by only a few seconds, there was little difference in the utility of learning either the win-stay or the win-shift strategy over perseverating in the same response pattern. Clearly, perseverating is a form of "stay" responding. The animal stays with the preceding response rather than the preceding platform location.

\section{EXPERIMENT 2 SUBMERSIBLE ESCAPE PLATFORM}

A second experiment comparing rats' acquisition of win-stay and win-shift escape responses was conducted under conditions that decreased the utility of persevera- 
tive responding and increased the utility of responding according to win-stay and win-shift strategies. Thus, the pool was equipped with two submersible escape platforms, which could be lowered following an incorrect first choice on any trial. Whenever a rat made an incorrect first choice, the escape platform was lowered to the bottom of the pool for $60 \mathrm{sec}$. This procedure forced the rats to swim at least $60 \mathrm{sec}$ longer after making an incorrect choice than after making a correct choice. Only by learning either the win-stay or the win-shift rule and remembering the information-trial position of the platform on the test trial could the rats consistently avoid being forced to swim an extra $60 \mathrm{sec}$. Since in the first experiment the win-shift group made fewer correct test-trial choices and more perseverative responses than the win-stay group, it was predicted that the win-shift group would be most affected by the forced delay in escape following incorrect choices.

\section{Method}

Subjects. Twenty naive rats, 10 in the win-stay and 10 in the win-shift group, were used in the second experiment.

Escape platform. The 21-cm-diameter plastic escape platform used in the first experiment was replaced by two submersible 19 $\mathrm{cm}$-diameter metal grid platforms. The platforms were painted white to make them less visible in the clouded water. The diameter of the grid was decreased to make chance encounters with the platform less likely, a change that would also seem to increase the utility of using a memory strategy as opposed to a perseveration strategy to perform the task. Attached to each platform were three pieces of fishing line, which were strung through three eyelets mounted on a wooden frame attached to the rim of the pool. Also, each platform was attached by a large rubber band to a piece of iron located on the bottom of the pool. The fishing line was used to raise the platforms at the appropriate time. The rubber band held the platform taut and forced the platform to the bottom of the tank when it was not being held in the elevated position. The three pieces of fishing line rising out of the water gave the rats cues to the two potential escape locations, but no information about whether a given platform was elevated.

Procedure. With the exception of two changes, the procedure of the second experiment was identical to the procedure of the first. One change involved lowering the escape platform for $60 \mathrm{sec}$ following an incorrect first choice on any trial. The second change involved the total number of pairs of trials given to the subjects and the time of day they were given. The subjects were given two pairs of trials per day for 5 days and three pairs of trials per day for the next 15 training days, or a total of 55 pairs of trials. On all training days pairs of trials were given at 0800 and $1730 \mathrm{~h}$. During the last 15 days the third daily pair of trials was given at $2100 \mathrm{~h}$.

\section{Results}

Figure 4 shows the mean number of correct responses made on both information and test trials across blocks of pairs of trials. The first block includes the first 5 days of training, during which the animals received 10 pairs of trials; each of the five subsequent blocks includes 3 days' training during which the animals received 9 pairs of trials. Thus, the scores for the first block were adjusted (multiplied by 0.9) to make them comparable to the scores for the last five blocks. A three-way mixed-factors ANOVA on these scores (task $\times$ trial type $\times$ block) revealed that all three main effects were significant: task $[F(1,18)=19.957, p<.001]$, trial type $[F(1,18)=$

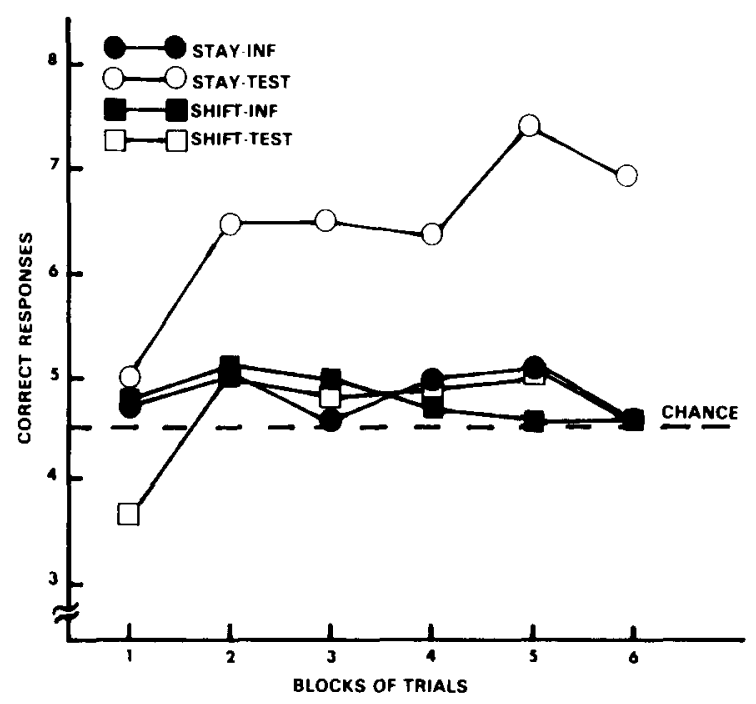

Figure 4. Mean number of correct responses made on information (INF) and test trials of win-stay and win-shin tasks across blocks of trials. The first block contsined 10 poirs of trials; the remaining blocks contained 9 pairs of trials. The means in the first block are adjusted accordingly. Note that 4.5 correct choices equals $50 \%$ correct responses or chance-level performance.

$32.608, p<.05]$, and block $[F(5,90)=3.440$, $p<.01]$. Also, the task $\times$ trial type $[F(1,18)=44.855$, $p<.001]$ and block $\times$ trial type $[F(5,90)=2.376$, $p<.05]$ interactions were significant. Post hoc examination of task $\times$ trial type means revealed that the win-stay group made more correct responses on test trials than they did on information trials and more than the win-shift group made on either information or test trials ( $p<.05$ in each case). Examination of Figure 4 reveals that neither group performed above chance $(>4.5)$ on information trials. The win-stay group did perform above chance over the last five blocks on test trials.

To determine whether the location of the platform on the test trial of one pair of trials affected the choice made on the subsequent information trial of the next pair of trials, information trial choices were examined. On information trials, neither win-stay nor win-shift groups chose the section that had contained the platform on the immediately preceding test trial at an above-chance level (52\% and $48 \%$, respectively). Clearly, on information trials both groups responded independently of where the platform had been placed on the preceding test trial.

Figure 5 shows the percentage of perseverative responses made by the win-stay and win-shift groups on information and test trials combined over blocks of trials. A two-way mixed-factors ANOVA (task $\times$ block) revealed significant task $[F(1,18)=14.627, p<.05]$ and block $[F(5,90)=5.376, p<.05]$ main effects. Thus, as is evident in Figure 5, the win-shift group perseverated more than the win-stay group, and both groups perseverated more over blocks of trials.

\section{Discussion}

The results of the second experiment confirm the results of the first. The win-stay rats learned the task, perform- 


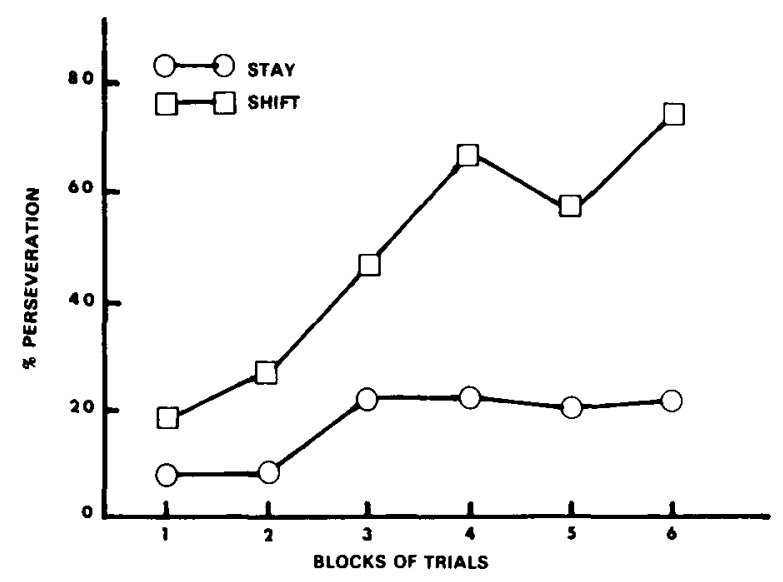

Figure 5. Mean percentage of perseverative responses made on win-stay and win-shint tecks serow blocks of trials. The first block contained 10 pairs of trinls; the other blocks contained 9 pairs of trials.

ing win-stay responses on test trials, and the win-shift animals did not learn the task, more often perseverating in their responses on both information and test trials. It is interesting that during the last block of trials the win-stay group made $76 \%$ win-stay responses on test trials, but only $52 \%$ win-stay responses on information trials. There are at least two possible explanations: (1) They may have been discriminating between information and test trials and using the win-stay strategy on test trials only when it was consistently reinforced; thus, choice behavior on information trials was not systematic. (2) They may have been attempting to use the win-stay strategy on all trials, but could not recall the previous test trial position of the platform on the information trials. Whatever the explanation, it is clear that the win-stay rats were using working memory to make choices on the test trials. The fact that the win-shift rats primarily made perseverative responses on both information and test trials suggests that they were using reference memory, responding in accord with their choices over several previous trials, not with the location of the platform on the preceding trial.

In addition to reaffirming the conclusions of the first experiment, the second experiment revealed that "sinking" the escape platform to force the rats to swim an extra $60 \mathrm{sec}$ following incorrect choices did not significantly alter performance of either win-stay or win-shift waterescape tasks. Examination of Table 1 reveals that the forced swimming resulted in neither an increase in correct choices nor a decrease in perseveration on either task. The imposed delay did not appear to predispose the rats to use a working memory strategy rather than a reference memory strategy, at least over the course of 55 pairs of trials.

Observation of the rats during the imposed delays revealed that they often swam over the locations of the escape platforms several times before the appropriate platform was raised. Thus, on a given trial they experienced several occasions on which the escape platform was not present in the position that was actually the correct location for that trial. Such misinformation may have made learning either the win-stay or win-shift strategies more difficult.

\section{EXPERIMENT 3 BARRIER}

A small number of subjects was used in a third experiment to determine whether the introduction of a barrier between the two platform positions would decrease perseverative responding and thus improve win-shift and win-stay performance. As with the imposed delay, it was felt that the barrier would decrease the utility of perseverating in the same response pattern, since it forced the rats to swim a longer route to reach the other platform.

\section{Method}

Subjects. Eight naive rats, 4 in the win-stay group and 4 in the win-shift group, were used in the third experiment.

Procedure. The subjects were given two pairs of trials per day, at approximately 0900 and $1800 \mathrm{~h}$, for 10 days-a total of 20 pairs of trials. On all trials a metal barrier was placed on the radius separating the left and right choice sections of the pool. Thus, following an incorrect choice a rat had to return to the starting section of the pool before going to the other choice section. The procedure of Experiment 3 was identical to that of Experiment 2 in all other respects. Thus, for both the win-shift and win-stay groups, incorrect choices were followed by both an imposed time delay and an increased swimming distance.

\section{Results}

The results of the barrier experiment are shown in Table 1 . Note that they are almost identical to the results

Table 1

Mean Number of Correct Choices and Perseverative Responses Made by Win-Stay and Win-Shif Groups on Information and Test Trials in Experiments 1, 2, and 3

\begin{tabular}{|c|c|c|c|c|c|c|c|c|c|c|c|}
\hline \multirow[b]{4}{*}{ Experiment/Condition } & \multicolumn{5}{|c|}{ Win-Stay } & \multicolumn{6}{|c|}{ Win-Shift } \\
\hline & \multicolumn{3}{|c|}{ Correct Choices } & \multirow{2}{*}{\multicolumn{2}{|c|}{ Perseverative }} & \multicolumn{4}{|c|}{ Correct Choices } & \multirow{2}{*}{\multicolumn{2}{|c|}{ Perseverative }} \\
\hline & Information & \multicolumn{2}{|c|}{ Test } & & & \multicolumn{2}{|c|}{ Information } & \multicolumn{2}{|c|}{ Test } & & \\
\hline & $\overline{M S E M}$ & & SEM & $M$ & $\overline{S E M}$ & $M$ & $\overline{S E M}$ & & $S E M$ & $M$ & $\overline{S E M}$ \\
\hline 1 No delay & $\begin{array}{ll}9.8 & 0.9\end{array}$ & 12.9 & 0.7 & 2.4 & 1.0 & 9.3 & 0.5 & 8.8 & 0.5 & 11.3 & 3.9 \\
\hline $260-\sec$ delay & $11.0 \quad 0.6$ & 12.8 & 0.6 & 2.5 & 0.9 & 10.9 & 0.5 & 9.5 & 0.8 & 7.6 & 1.8 \\
\hline $\begin{array}{l}360 \text {-sec delay } \\
+ \text { barrier }\end{array}$ & $10.0 \quad 0.6$ & 13.2 & 1.6 & 5.8 & 1.8 & 10.0 & 1.1 & 9.2 & 0.5 & 16.5 & 5.1 \\
\hline
\end{tabular}

Note-Data for Experiment 2 include first 20 pairs of trials only. 
of the first two experiments. The win-stay group made more correct choices on test trials and fewer perseverative responses on all trials than did the win-shift group. A two-way mixed-factors ANOVA (task $\times$ trial type) on the total number of correct responses made on all test trials revealed a nearly significant interaction $[F(1,6)=4.861$, $p=.07]$. The main effects were not significant. The difference in number of perseverative responses was not significant $[t(6)=1.73, p>.05]$.

\section{Discussion}

A comparison of the results of Experiment 3 with those of both Experiment 1 and the first 20 pairs of trials of Experiment 2 (see Table 1) clearly reveals that the barrier had little or no effect on choice behavior. The barrier did not alter the relative performance levels on the two tasks or the amount of perseverative responding.

The results of the three experiments are remarkably similar. Collectively, they demonstrate that rats acquire a win-stay escape response much more readily than they acquire a win-shift escape response. Furthermore, rats that fail to acquire either the win-stay or the win-shift response pattern tend to develop a position habit, repeatedly making the same first choice on trials. Apparently, the rat has a strong innate tendency to repeat the same response in attempting to escape from water, even if it is not the most direct route.

\section{EXPERIMENT 4 RESPONSE PREFERENCE}

The fourth experiment was designed to determine what response pattern rats would develop in the water escape task if both win-stay and win-shift responses were reinforced. Given that win-stay is more readily acquired, it was predicted that the rats would be more likely to develop a win-stay than a win-shift pattern. To minimize perseverative responding, a procedure was introduced to discourage perseverative responses.

\section{Method}

Subjects. Ten naive subjects were used.

Procedure. The procedure used in the response preference experiment was similar to that of Experiment 1 except for two major differences. First, in Experiment 4 there were two plastic escape platforms, one at each escape location, present on all test trials. Thus, on the information trials a platform was placed in either the left or the right choice section, following a Gellermann-type sequence, and on test trials platforms were located in both positions. Second, whenever a rat began to perseverate in its choice response (i.e., when it had made six consecutive responses to the same position on information and test trials), the opposite section was made the correct choice on subsequent information trials until the rat finally went to that section first on any trial. All subjects received two pairs of trials per day, at 1200 and $2000 \mathrm{~h}$, for 5 days and three pairs of trials per day at 1200,1600 , and $2000 \mathrm{~h}$ for 15 days-a total of 55 pairs of trials.

\section{Results}

Figure 6 shows the percentage of win-stay and win-shift responses on test trials and the percentage of

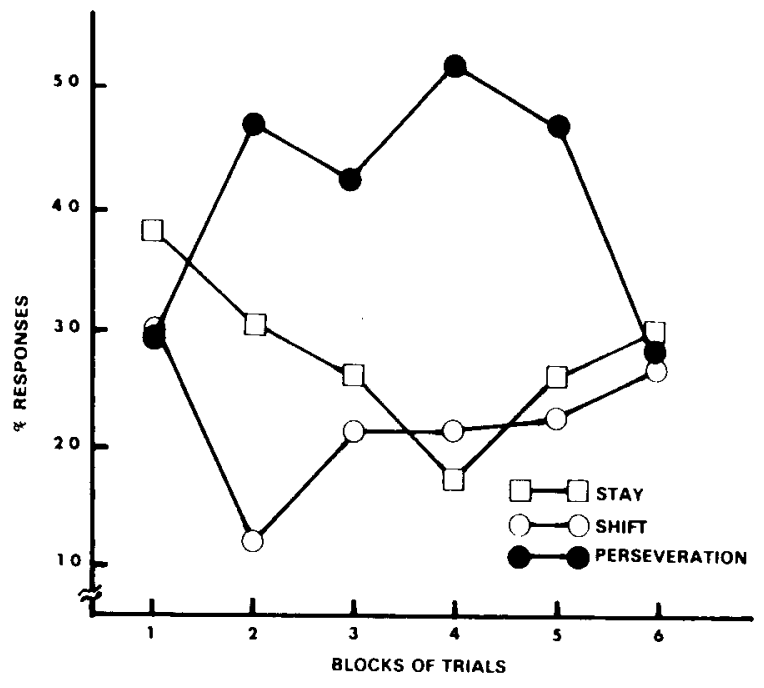

Figure 6. Mean percentage of win-stay, win-shif, and perseverative responses made across blocks of trials. The first block contained 10 pairs of trials; the others contained 9 pairs of trials.

perseverative responses (more than six consecutive first choices to the same escape section) on both information and test trials across blocks. Whenever the procedure to correct perseverative responses was in effect for a given rat, any perseverative response made on a test trial was also a win-shift response; thus, to avoid inflating the win-shift response scores, a given response was scored in only one category-any response scored as a perseverative response was not also counted as a win-shift response. A two-way repeated factors ANOVA (response type $\times$ block) on percentage of win-stay and win-shift responses on test trials showed neither a significant interaction nor any significant main effects. Also, a oneway repeated measures ANOVA on the percentage of perseverative responses across blocks on all trials failed to show any significant differences. Both analyses revealed that there was much within-cell variability in the scores of the subjects.

Examination of the behavior of individual rats over trials revealed a noticeably similar response pattern across subjects. Each of the rats would first make a short series of responses with no apparent strategy or bias. This series would include both win-stay and win-shift responses. Next, each subject would begin to perseverate in its choices, always going to the same section first. After six consecutive same-side first choices occurred, the perseveration correction procedure was initiated and the rat would eventually enter the opposite section first. At this time the correction procedure was discontinued and the rat would either immediately begin perseverating again or make a few unpredictable responses and then begin perseverating again. Each rat repeated this cycle several times. During any given block of trials several rats would be perseverating and several would be making a combination of win-stay and win-shift responses. Thus, the analyses of win-stay, win-shift, and perseverative responses across blocks showed large within-cell variance and no significant effects. 


\section{Discussion}

The results of the response preference experiment clearly show that in a free-choice two-alternative water escape situation in which win-stay, win-shift, and perseverative responding all result in the same percentage of reinforcement (50\% on information trials and $100 \%$ on test trials, or $75 \%$ over all trials), rats develop a perseverative response strategy. Only when the perseverative response results in consistent repeated nonreinforcement do the animals stop repeating the same response. Having extinguished, the animals again begin to perseverate in making one choice after only a few trials, never showing a consistent win-stay or win-shift pattern that is related to the platform's location on the preceding trial. Since all three response patterns result in the same percentage of reinforcement, the perseverative responding appears to reflect an unlearned response bias that affects behavior in a water escape situation.

\section{GENERAL DISCUSSION}

Collectively, the four experiments suggest that the most natural response of rats given pairs of free-choice trials in a water maze is to perseverate. They adopt a course that efficiently takes them to both potential escape platform locations. Such a strategy results in relatively short escape latencies and the same first choice from trial to trial. This is particularly true when all response strategies-win-stay, win-shift, and perseveration-are equally reinforced, as in Experiment 4 . In following the same path on every trial the animals appear to be using a reference memory strategy. The immediately preceding trial is used only to the extent that it influences the collective memory of all preceding trials. The first three experiments show that rats can learn to respond according to a working memory win-stay strategy when such a strategy results in more frequent reinforcement than does perseverative responding. None of the experiments provides evidence that rats can learn to respond according to a working memory win-shift strategy in the water maze when it results in more frequent reinforcement than the reference memory perseveration strategy. Although it is possible that the rats could eventually acquire a win-shift response if enough pairs of trials were given, the increasing perseveration of the win-shift group across blocks of pairs of trials (see Figures 3 and 5) suggests that perseveration only gets stronger with more trials. This is true even when a forced delay following incorrect choices and a barrier are added to reduce the utility of perseveration. The failure of the rats to acquire a win-shift response is not due to the win-shift task's being insoluble or harder to learn than win-stay, per se, since in exploratory and foodand water-reinforced situations rodents acquire win-shift responses more readily than win-stay responses.

Collectively, the four experiments suggest that in a twochoice water escape situation two unlearned biases influence the response strategy used by rats. First, in situations in which reference and working memory strategies are equally effective, the reference memory strategy is adopted. Thus, in the response preference experiment, in which all three response strategies resulted in an equal probability of escape, all subjects used the reference memory perseveration strategy until the response perseveration correction procedure was instituted. Second, in situations in which working memory strategies result in greater probability of escape than does the reference memory perseveration strategy, as in the first three experiments, animals have a bias to repeat successful escape responses (win-stay). Thus, they use the working memory strategy only if it involves win-stay. Apparently, rats can supersede one response tendency, that is, responding according to reference memory, but they cannot readily acquire a response pattern that involves superseding two response tendencies, that is, using reference memory and making win-stay choices.

The results of the present study, although somewhat surprising, are not inconsistent with some earlier observations. The author, in testing rats for spontaneous alternation in a T-maze, has noted many times that if anything unusual happens to disturb an animal on a given test trial, such as the experimenter's bumping the maze or dropping something or the rat's accidentally getting pinched in the maze, the rat will not alternate on that trial even if its usual rate of spontaneous alternation is above $80 \%$. Also, recall that it has been demonstrated that shocking mice in the startbox, choice point, or choice alley results in perseverative responding (Mitchell et al., 1984). Finally, animals are often found to perseverate in active avoidance tasks, being extremely resistant to extinction (Sheffield \& Temmer, 1950; Solomon, Kamin, \& Wynne, 1953). Whether it is due to heightened arousal level or an inherited response bias or both, response perseveration seems to be the natural response in the water escape situation in particular, and probably in escape situations in general.

\section{REFERENCES}

Berlyne, D. P. (1960). Conflict, arousal and curiosity. New York: McGraw-Hill.

Burešovi, O., Bureš, J., Ottzl, M. S., Z Zahalka, A. (1985). Radial maze in the water tank: An aversively motivated spatial working memory task. Physiology \& Behavior, 34 1003-1005

Demrer, W. N., \& EARL, R. W. (1957). Analysis of exploratory, manipulatory, and curiosity behavior. Psychological Review, 64, 91-96.

Douglas, R. J. (1966). Cues for spontaneous alternation. Journal of Comparative \& Physiological Psychology, 62, 171-183.

Gaffan, E. A., DAvies, J. (1981). The role of exploration in win-shift and win-stay performance on a radial maze. Learning \& Motivation, 12, 282-299.

GellermanN, L. W. (1933). Chance orders of alternating stimuli in visual discrimination experiments. Journal of Genetic Psychology, 42, 206-208.

Glanzer, M. (1958). Curiosity, exploratory drive, and stimulus satiation. Psychological Bulletin, 55, 302-315.

Mactutus, C. F., MuRray, D. L. (1986). Spatial memory: Win-stay vs. win-shift strategies. Society for Neuroscience Abstracts, 12, 520 . Mrans, L. W., Harrell, T. H., Mayo, E. S., * AleXander, G. B. (1974). Effects of dorsomedial thalamic lesions on spontaneous al 
ternation, maze activity and runway performance in the rat. Physiology \& Behavior, 12, 973-979.

Means, L. W., Leander, J. D., Isaacson, R. L. (1971). The effects of hippocampectomy on alternation behavior and response to novelty. Physiology \& Behavior, 6, 17-22.

Mitchell, D., Koleszar, A. S., \& SCopatz, R. A. (1984). Arousal and $\mathrm{T}$-maze choice behavior in mice: $\mathrm{A}$ convergent paradigm for neophobia constnucts and optimal arousal. Learning \& Motivation. 15, 287-301.

MoRRIS, R. G. M. (1981). Spatial localisation does not depend on the presence of local cues. Leaming \& Motivation, 12, 239-260.

MoRRIs, R. (1984). Developments of a water-maze procedure for studying spatial learning in the rat. Joumal of Neuroscience Methods, 11, 47-60.

Olton, D. S., \& Coluson, C. (1979). Intramaze cues and "odor trails" fail to direct choice behavior on an elevated maze. Animal Learning \& Behavior, 7, 221-223.

Olton, D. S., Handelmann, G. E., \& Walker, J. A. (1981). Spatial memory and food searching strategies. In A. C. Kamil \& T. Sargent (Eds.), Foraging behavior: Ecological, ethological and psychological approaches. New York: Garland STPM Press.

Olton, D. S., \& SChlosberg, P. (1978). Food-searching strategies in young rats: Win-shift predominates over win-stay. Journal of Comparative \& Physiological Psychology, 92, 609-618.

Sheffield, F. D., Temmer, H. W. (1950). Relative resistance to extinction of escape training and avoidance training. Journal of $E x$ perimental Psychology, 40, 287-298.

Solomon, R. L., Kamin, K. J., WynNe, L. C. (1953). Traumatic avoidance learning: The outcomes of several extinction procedures with dogs. Joumal of Abnormal Social Psychology, 48, 291-302.

Stanton, M. E., Thomas, G. J., \& BRto, G. N. (1984). Posterodorsal septal lesions impair performance on both shift and stay working memory tasks. Behavioral Neuroscience, 3, 405-415.

Sutherland, R. J., LingGard, R. (1982). Being there: A novel demonstration of latent spatial learning in the rat. Behavioral \& Neural Biology, 36, 103-107.

Sutherland, R. J., Whishaw, I. Q., \& Kolb, B. (1983). A behavioural analysis of spatial localization following electolytic, kainate- or colchicine-induced damage to the hippocampal formation in the rat. Behavioural Brain Research, 7, 133-153.

(Manuscript received October 19, 1987; revision accepted for publication January 28, 1988.) 